Research Article

\title{
A Giant Magnetoimpedance-Based Microfluidic System for Multiplex Immunological Assay
}

\author{
Shan Gao ${ }^{1}$, Lin Kang ${ }^{1}$, Min Deng ${ }^{2}$, Bin $\mathrm{Ji}^{1}$, Jing Liu ${ }^{1}$, Wenwen Xin ${ }^{1}$, Jingjing Kang ${ }^{1}$, Ping $\mathrm{Li}^{1}$, Jie \\ $\mathrm{Gao}^{1}$, Jinglin Wang ${ }^{1}$, Hao Yang ${ }^{1}$ \\ ${ }^{1}$ State Key Laboratory of Pathogen and Biosecurity, Institute of Microbiology and Epidemiology, No. 21 Dongda Street, Beijing \\ 100071, China. \\ ${ }^{2}$ Department of Nano Biomedicine and Engineering, Key Laboratory for Thin Film and Microfabrication of Ministry of Education, \\ Institute of Micro/Nano Science and Technology, Shanghai Jiao Tong University, 800 Dong Chuan Road, Shanghai 200240, China. \\ ${ }^{\dagger}$ Shan Gao, Lin Kang and Min Deng contributed equally to this work. \\ Corresponding authors. E-mail: wjlwj10801@sina.com; tohaoyang@hotmail.com
}

Received: Oct. 16, 2016; Accepted: Nov. 10, 2016; Published: Nov. 23, 2016.

Citation: Shan Gao, Lin Kang, Min Deng, Bin Ji, Jing Liu, Wenwen Xin, Jingjing Kang, Ping Li, Jie Gao, Jinglin Wang, and Hao Yang, A Giant Magnetoimpedance-Based Microfluidic System for Multiplex Immunological Assay. Nano Biomed. Eng., 20 16, 8(4): 240-245.

DOI: $10.5101 /$ nbe.v8i4.p240-245.

\begin{abstract}
Microfluidic chip and giant magnetoimpedance (GMI)-related technology has developed quickly over the past decades in the field of biological detection. In this work, we designed and fabricated a GMIbased microfluidic system for screening of multiplex gastric cancer biomarkers. The microfluidic chip and GMI sensor were prepared by micro-electromechanical systems (MEMS) technology. This system can analyse 8 gastric cancer protein biomarkers simultaneously in less than 25 mins and offer more stable detection signal than conventional enzymological or fluorescent methods. The microfluidic chip was then tested in 150 clinical specimens and compared with enzyme-linked immunosorbent assay (ELISA) method. The results indicated no significant difference and excellent agreement. In short, the prototype of GMI-based microfluidic system has been developed successfully and showed promising potentials for parallel screening of cancer biomarkers.
\end{abstract}

Keywords: Microfluidic chip; GMI; MEMS; Immunological assay

\section{Introduction}

Microfluidic chip-related technology has developed quickly over the past decades in the field of biological detection. It integrates multiple laboratory techniques in a chip with a footprint of at most a few tens of square centimeters; therefore, a lot of laborious and repetitive work can be saved. In addition, microfluidic devices offer shorter detection time and require minimal amounts of sample. Because the sample is handled in a sealed environment, the risk of cross- contamination is also reduced significantly. So far, various microfluidic devices have been fabricated and proved to be useful for enhancing the efficiency of bioassays and medical diagnosis [1-5].

Giant magnetoimpedance (GMI) effect is the change of the impedance experienced by an $\mathrm{AC}$ flowing through a soft magnetic material when an external DC magnetic field is applied [6]. It has the advantages of high sensitivity, quick response, low cost, easy-touse and high operational stability, which can be used to fabricate high performance microfluidic analytical 
system. In the recent years, some GMI-based sensors have been developed in a variety of forms including magnetic film, ribbon and microwire [7]. Several prototype of GMI-biosensors have also been developed for the detection of nucleic acid, cancer cell, bacterium and so on $[1,7,8]$.

The aim of this work is to develop a prototype of GMI-based microfluidic system for the screening of multiplex gastric cancer biomarkers. The GMI sensor based on Metglas ${ }^{\circledR}$ 2705M ribbon was fabricated by micro-electromechanical systems (MEMS) technology according to our previously published work [1, 7]. Functionalized magnetic nanoclusters (MNCs) were designed by coating carboxyl MNCs with eight antibodies respectively. Eight different paired antibodies were immobilized on different detection areas in the microfluidic chip. The GMI responses for clinical serum samples were measured and the resulted data were compared with traditional enzyme linked immunosorbent assay (ELISA).

\section{Materials and Methods \\ Materials}

Ethylene glycol, anhydrous ferric chloride, citric acid, anhydrous sodium acetate, polyvinya alcohol (PVA), Isopropyl-1-thio- $\beta$-d-galactopyranoside (IPTG), sodium borohydride, ethylene glycol (EG) and carbodiimide hydrochloride (EDC) were obtained from Sigma. Bovine serum albumin (BSA) and 2-[N-morpholino] ethanesulfonic acid (MES) was purchased from Shanghai MajorBio Technologies Co., Ltd. (Shanghai, China). The ELISA kits, protein standard and paired antibodies of eight caner biomarkers (CA199, CEA, CA125, VEGF, Gastrin 17, CA724, PGI and PGII) were purchased from Abcam plc or donated by Dr. Ding Li of No. 261 Hospital of PLA (People's Liberation Army). In all experiments, deionized water was used. 150 specimens from the outpatients for gastric cancer screening were collected in the Department of Gastroenterology of No. 261 Hospital of PLA. All work with human serum was approved by Ethics Committee of both Shanghai Jiao Tong University and No. 261 Hospital of PLA.

\section{Preparation of functional MNCs}

The preparation of carboxyl MNCs was carried out by previous methods published elsewhere [9]. In brief, $0.12 \mathrm{M} \mathrm{FeCl}_{3} \cdot 6 \mathrm{H}_{2} \mathrm{O}, 0.44 \mathrm{M}$ anhydrous sodium acetate and $0.021 \mathrm{M}$ citric acid were well dissolved in $35 \mathrm{~mL}$ EG under ultrasonication. The solution was then sealed in a $50 \mathrm{~mL}$ Teflon lined stainless-steel autoclave and heated at $200{ }^{\circ} \mathrm{C}$ for $10 \mathrm{hrs}$. After cooling down, the black sediment was separated magnetically and washed with ethanol and deionized water for 3 times respectively to eliminate organic and inorganic impurities, and then dried in a vacuum at $60{ }^{\circ} \mathrm{C}$. A JEOL2010 transmission electron microscope (TEM) and JEOL scanning electron microscope (SEM) were used for taking images of MNCs. Magnetization of MNC powders was carried out on a Lakeshore 7300 vibration sample magnetometer (VSM).

To couple MNCs with primary antibodies, $10 \mathrm{~mL}$ of $1 \mathrm{mg} / \mathrm{mL}$ MNCs and $10 \mathrm{~mL}$ of $1 \mathrm{mg} / \mathrm{mL}$ primary antibodies against different cancer biomarkers were mixed respectively, and then $1 \mathrm{~mL}$ of $1 \mathrm{mg} / \mathrm{mL}$ EDC was added and blended by pipetting up and down. The mixture reacted under continuous rotation in roller mixer at room temperature for $3 \mathrm{hrs}$ and was then separated magnetically. BSA was added into the solution at a concentration of $1 \mathrm{mg} / \mathrm{mL}$ and incubated at room temperature for $3 \mathrm{hrs}$ to block potentially unreacted surface of MNCs. The functional MNCs were then separated magnetically and the supernatant was discarded. $10 \mathrm{~mL}$ PBS [Phosphate-Buffered Saline (PBS); $0.2 \mathrm{mg} / \mathrm{mL} \mathrm{KCl,} 1.44 \mathrm{mg} / \mathrm{mL} \mathrm{Na}_{2} \mathrm{HPO}_{4}, 0.24$ $\left.\mathrm{mg} / \mathrm{mL} \mathrm{KH}_{2} \mathrm{PO}_{4}, 8 \mathrm{mg} / \mathrm{mL} \mathrm{NaCl}, \mathrm{pH} 7.4\right]$ with $0.5 \%$ tween-20 (v/v) and 1\% BSA was used to resuspend and wash the functional MNCs for 3 times. Finally, the functional MNCs were dispersed in $10 \mathrm{~mL}$ PBS with $0.5 \%$ tween-20 (v/v) and $0.5 \%$ BSA and kept at $4{ }^{\circ} \mathrm{C}$ until further use.

\section{GMI-based microfluidic system}

GMI biosensor is fabricated with soft magnetic ribbon material (Metaglas $\AA 2705 \mathrm{M}$ ) according to our published methods [7]. The GMI curves were measured by an impedance analyzer. The ac current flows through the sensor with a constant current amplitude of $10 \mathrm{~mA}$. The maximum absolute value of external magnetic field (Hex) was 150 Oe and applied along the longitudinal direction of the sensor as shown in (Fig. 1). The GMI ratio is calculated from $Z(H)$ curves defined as: GMI ratio $=100 \% \times\left[Z(H)-Z\left(H_{0}\right)\right] /$ $Z\left(H_{0}\right)$, where $Z(H)$ is the magnetoimpedance with $0-150$ Oe magnetic field. The working range of GMI sensor, where the maximum GMI ratio located, stay in the higher fields range (around $15 \mathrm{Oe}$ ).

Microchannel was prepared by wet etching of a 


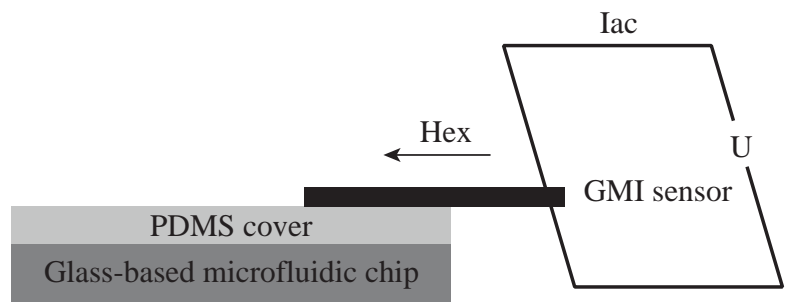

Fig. 1 Detection principle of GMI biosensor.

Pyrex glass with the thickness of $2 \mathrm{~mm}$. The depth and width of microchannel were 100 and $600 \mu \mathrm{m}$, with a $400 \mu \mathrm{m}$ space between channels in detection region. $1 \mathrm{~mm}$ Polydimethylsiloxane (PDMS) cover was adopted to encapsulate microfluidic chip. Eight different capture antibodies were immobilized on the surface of microchannel in eight detection regions as showed in Fig. 2. The surface modification of glass microchannel was the same as Ref. [10]. And immobilization of capture antibodies was through traditional EDC chemistry.

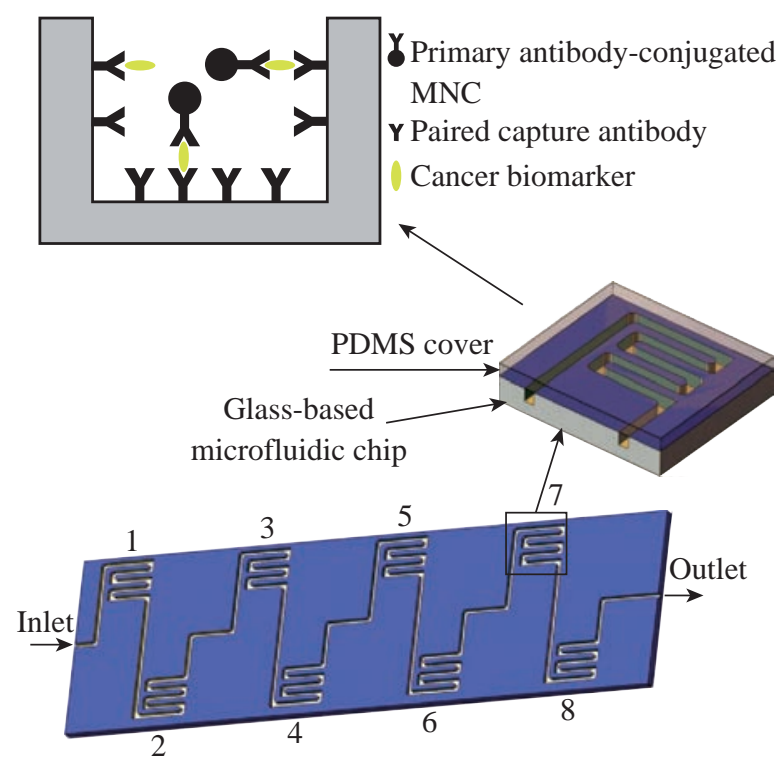

Fig. 2 The schematic illustration of GMI-based microfluidic chip (detection area 1: CA199; 2: CEA; 3: CA125; 4: VEGF; 5: Gastrin 17; 6: CA724; 7: PGI; 8: PGII).

\section{Analysis of clinical samples}

To analyse the samples, eight primary antibodiesfunctinalized MNCs were blended in equal proportion thoroughly, $80 \mu \mathrm{L}$ as-prepared suspension was mixed and incubated with $100 \mu \mathrm{L}$ sample at $37{ }^{\circ} \mathrm{C}$ for 10 mins. After the free cancer biomarkers in serum were captured by the primary antibody on MNCs, magnetic separation was carried out. $1 \mathrm{~mL}$ PBST (PBS with $0.5 \%$ Tween 20 ) was used to resuspend the MNCs. The resuspension was injected into microchannel from the inlet at $100 \mu \mathrm{L} / \mathrm{min}$ flow rate at $37{ }^{\circ} \mathrm{C}$ with a syringe pump for specific capture. After the biomarkersfunctional MNC complexes were captured by the paired primary antibody on the microchannel of corresponding detetion areas, the microchannel was then washed twice by PBST and used for analysis by GMI biosensor. In this work, eight gastric cancer biomarker standards were spiked in $100 \mu \mathrm{L}$ of PBS at the recognized or empirical cut-off concentrations to set up the cut-off value of GMI ratio as follows: cut-off value $=$ average maximum GMI ratio of ten biomarker standard - spiked PBS $+2 \times$ standard deviation. ELISA was used for comparison with the same clinical samples. The performance of the two methods was analyzed with Statistics Package for Social Sciences (SPSS) software (SPSS Inc, Chicago, USA).

\section{Results and Discussion}

The MNC was prepared by a traditional solvothermal reaction at $200{ }^{\circ} \mathrm{C} . \mathrm{FeCl}_{3} \cdot 6 \mathrm{H}_{2} \mathrm{O}$ was deoxidated by $\mathrm{EG}$ in the presence of sodium acetate as alkali source, biocompatible citric acid and PVA as stabilizer. The excess EG acted as both the solvent and the reductant. Typically, the as-prepared MNC was approximately $200 \mathrm{~nm}$ in diameter. The TEM and SEM images of the as-prepared MNC are shown in Fig. 3(a) and (b), which demonstrate that the clusters were nearly spherical and uniform in size. Each cluster was composed of many magnetic nanocrystals. The data of magnetic properties indicated that the MNC exhibited superparamagnetic behavior with a saturation moment of around $44 \mathrm{emu} / \mathrm{g}$ at $300 \mathrm{~K}$, as shown in Fig. 4. Due to the electrostatic repulsion and steric hindrance effect, the MNC can be easily dispersed in PBS or other biocompatible solution, which favors their biomedical application. The fabricated glass-based microfluidic chip was observed by optical microscope and SEM for thedetailed structure of microchennel (Fig. 5 (a) and (c)). The rough surface is typical for the fabrication of glass-based materials by the wet etching.

The GMI-based microfluidic system is based on three interacting magnetic fields: the ac magnetic field of the ac current passing through the sensor element, the dc external magnetic field produced by a coil and the induced magnetic fields of the MNC [7]. GMI sensor was used to detect the induced magnetic fields of MNCs-conjugated primary antibody-cancer biomarker complexes captured by specific paired primay antibody on the surface of microchannel in 
corresponding detection regions. Each sample was tested in three manners and average GMI ratio was taken as the final result. Therefore, the risk status of
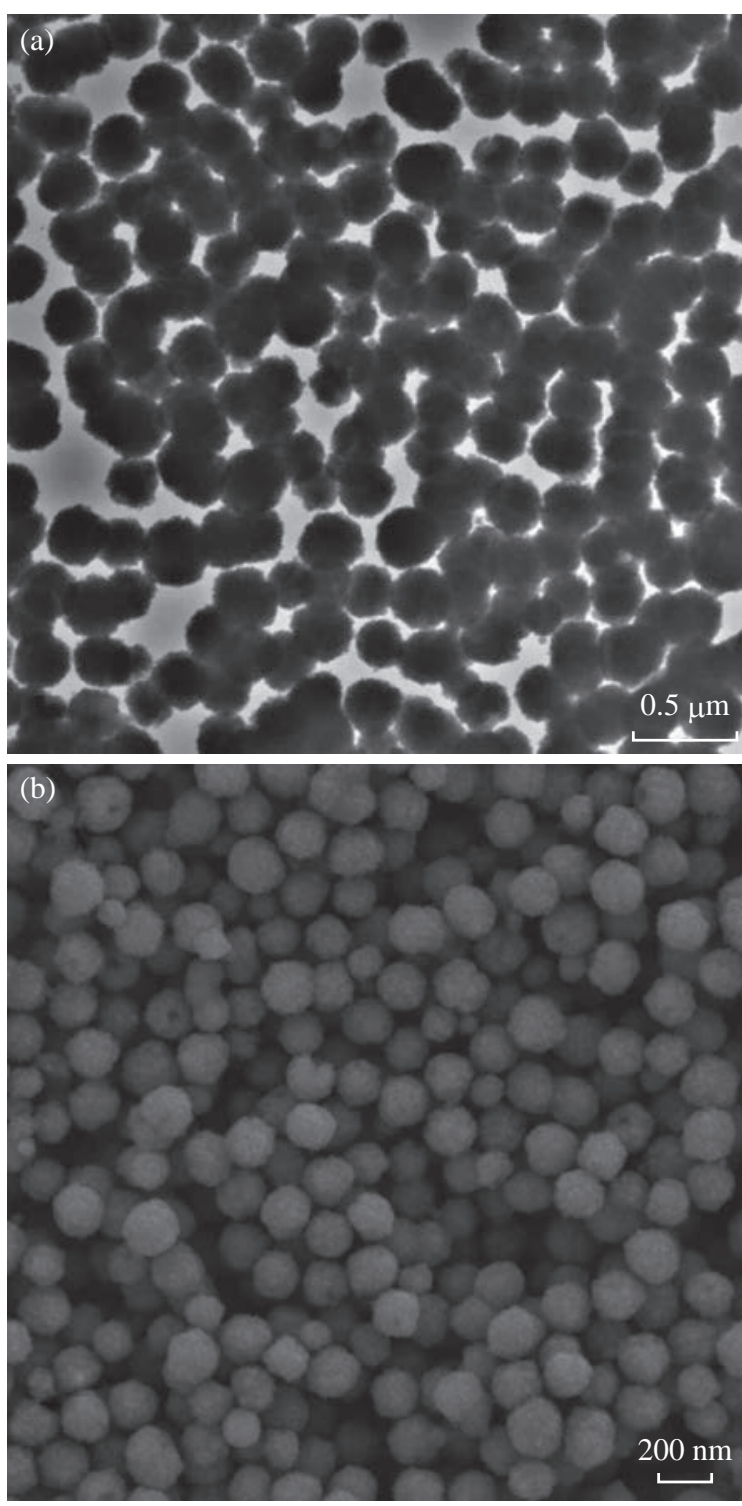

Fig. 3 (a) TEM and (b) SEM figures of MNC.

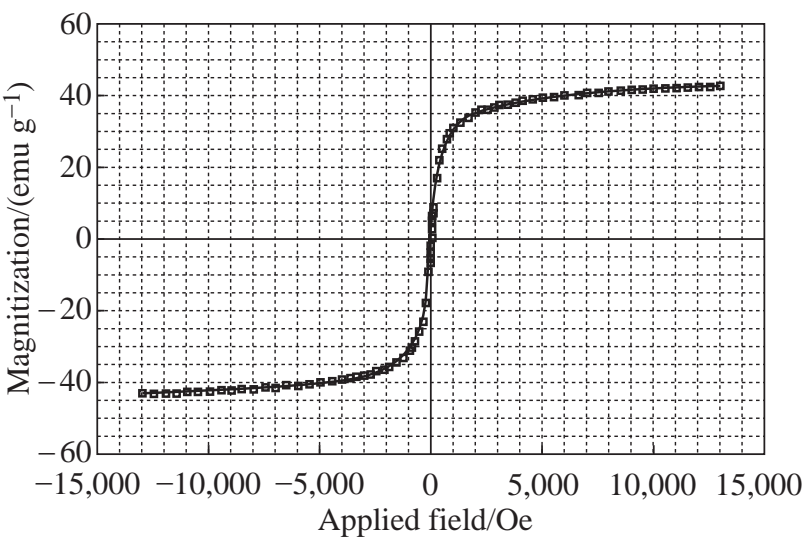

Fig. 4 Magnetization curves of MNCs powders measured at $300 \mathrm{~K}$.

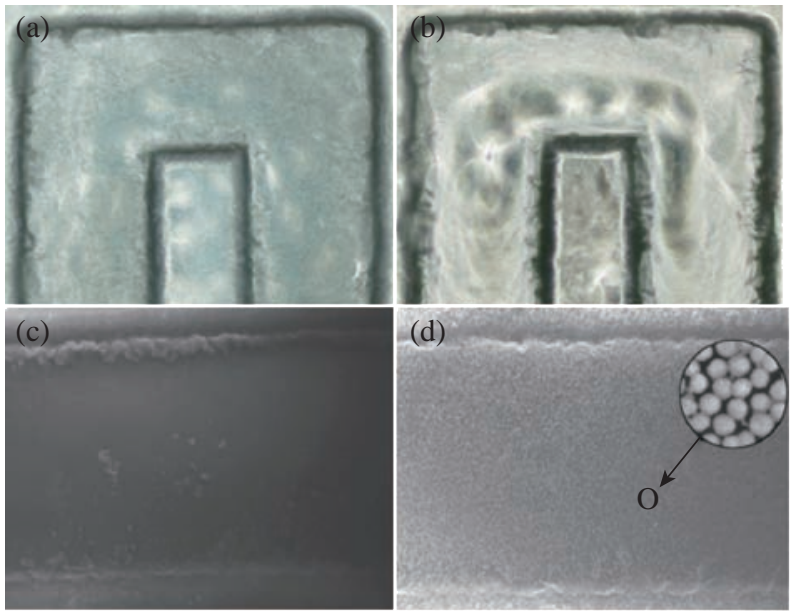

Fig. 5 Optical microscope (OM) and SEM figures of typical negative results and positive results: (a) OM-negative; (b) OMpositive; (c) SEM-negative; and (d) SEM-positive.

eight gastric cancer biomarkers can be determined parallelly by the GMI curve changes in corresponding detection region after capture.

In this work, 8 gastric cancer-associated biomarkers (CA19-9, CEA, CA125, VEGF, Gastrin 17, CA72-4, PGI and PGII) were empirically chosen for the proofof-concept through the multiplex immunological assay in GMI-based microfluidic chip. Among these cancer biomarkers, CA19-9, CEA and CA72-4 are the most routinely used predictors for gastric cancer [11]. However, only the three predictors mentioned above could not fully meet the requiements for the early detection or screening of gastric cancer. The joint detection with multiplex biomarkers can significantly improve the positive rate [12]. For this reason, five other gastric cancer-related biomarkers (CA125, VEGF, Gastrin 17, PGI and PGII) were also incorporated into the serum biomarker panel for the potential risk evalutation of gastric cancer [1315]. At present, ELISA or chemoluminescence are the most common methods used for the screening of these biomarkers in serum. But the two methods suffer from the same problem: laborious and repetitive work as well as unparallel multiplex detetion, which tremendously lowers the efficiency of screening. Hence, we designed this GMI-based microfluidic chip for the proof-of-concept of multiplex immunological assay. The cut-off point of these biomarkers were set up according to the published literatures or clinical experience as follows: CA19-9 $(\leq 37 \mathrm{U} / \mathrm{mL})$, CEA $(\leq$ $5 \mu \mathrm{g} / \mathrm{L}), \mathrm{CA} 125(\leq 35 \mathrm{U} / \mathrm{mL}), \operatorname{VEGF}(\leq 142.2 \mathrm{pg} / \mathrm{mL})$, Gastrin $17(\leq 2 \mathrm{pmol} / \mathrm{L}), \mathrm{CA} 72-4(\leq 6 \mathrm{U} / \mathrm{mL})$, PGI $(<25 \mu \mathrm{g} / \mathrm{L})$ and PGII $(\mathrm{PGI} / \mathrm{PGII}<2.5)$ [11-15]. 150 
Table 1 The comparison between ELISA and microfluidic chip

\begin{tabular}{|c|c|c|c|c|c|c|}
\hline \multirow{2}{*}{\multicolumn{2}{|c|}{ Microfluidic chip }} & \multicolumn{2}{|c|}{ ELISA } & \multirow{2}{*}{ Total } & \multirow{2}{*}{$p$ value by McNemar Test } & \multirow{2}{*}{ Kappa value } \\
\hline & & $\geq$ cut-off point & $<$ cut-off point & & & \\
\hline \multirow{3}{*}{ CA19-9 } & $\geq$ cut-off point & 107 & 0 & 107 & \multirow{3}{*}{$>0.05$} & \multirow{3}{*}{0.967} \\
\hline & $<$ cut-off point & 2 & 41 & 43 & & \\
\hline & Total & 109 & 41 & 150 & & \\
\hline \multirow{3}{*}{ CEA } & $\geq$ cut-off point & 98 & 1 & 99 & \multirow{3}{*}{$>0.05$} & \multirow{3}{*}{0.970} \\
\hline & $<$ cut-off point & 1 & 50 & 51 & & \\
\hline & Total & 99 & 51 & 150 & & \\
\hline \multirow{3}{*}{ CA125 } & $\geq$ cut-off point & 105 & 0 & 105 & \multirow{3}{*}{$>0.05$} & \multirow{3}{*}{0.984} \\
\hline & $<$ cut-off point & 1 & 44 & 45 & & \\
\hline & Total & 106 & 44 & 150 & & \\
\hline \multirow{3}{*}{ VEGF } & $\geq$ cut-off point & 99 & 0 & 99 & \multirow{3}{*}{$>0.05$} & \multirow{3}{*}{1} \\
\hline & $<$ cut-off point & 0 & 51 & 51 & & \\
\hline & Total & 99 & 51 & 150 & & \\
\hline \multirow{3}{*}{ Gastrin 17} & $\geq$ cut-off point & 3 & 0 & 3 & \multirow{3}{*}{$>0.05$} & \multirow{3}{*}{1} \\
\hline & $<$ cut-off point & 0 & 147 & 147 & & \\
\hline & Total & 3 & 147 & 150 & & \\
\hline \multirow{3}{*}{ CA72-4 } & $\geq$ cut-off point & 83 & 1 & 84 & \multirow{3}{*}{$>0.05$} & \multirow{3}{*}{0.973} \\
\hline & $<$ cut-off point & 1 & 65 & 66 & & \\
\hline & Total & 84 & 66 & 150 & & \\
\hline \multirow{3}{*}{ PGI* } & $\geq$ cut-off point & 0 & 0 & 0 & \multirow{3}{*}{-} & \multirow{3}{*}{-} \\
\hline & $<$ cut-off point & 0 & 150 & 150 & & \\
\hline & Total & 0 & 150 & 150 & & \\
\hline \multirow{3}{*}{ PGI/PGII } & $\geq$ cut-off point & 1 & 0 & 1 & \multirow{3}{*}{$>0.05$} & \multirow{3}{*}{1} \\
\hline & $<$ cut-off point & 0 & 149 & 149 & & \\
\hline & Total & 1 & 149 & 150 & & \\
\hline
\end{tabular}

$p>0.05$, there was no significant difference between the two methods

*No statistics were computed because Microfluidic and ELISA are constants.

random sera from the outpatients were analyzed with both the microfluidic chip and the commercial ELISA kit. The test results showed that there was no significant difference between the two methods (Table 1). The microfluidic chip and ELISA indicateed excellent agreement with the kappa values higher than 0.9 (Table 1). Compared with ELISA format, the GMI-based microfluidic chip shows several unique advantages. The first one is time saving. Its overall operation time is less than 25 mins. Secondly, it has the merit of multiplex detection. 8 gastric cancer biomarkers can be analysed simultaneously. Last but not the least, the stability of detection signal from MNC-labelled probes is significantly higher than conventional enzymological or fluorescent signal.

\section{Conclusions}

In this work, we designed and developed a GMI-based microfluidic system for screening of multiplex gastric cancer biomarkers. The microfluidic chip and GMI sensor were fabricated by MEMS technology. This system can analyse 8 gastric cancer protein biomarkers simultaneously in less than 25 mins and offer more stable detection signal than conventional enzymological or fluorescent methods. The microfluidic chip was then tested in 150 clinical specimens and compared with ELISA method. The results indicated no significant difference and excellent agreement. All in all, the prototype of GMI-based microfluidic system has been developed successfully 
and showed promising potentials for screening of cancer biomarkers.

\section{Acknowledgements}

This work was supported by project SKLPBS1403, 14QNP117 and AWS15J006, and the National Key Basic Research Program (973 Project) (No. 2015CB931802), National Natural Scientific Fund (No. 81225010, 81327002, and 31100717), 863 Project of China (2014AA020700), Shanghai Science and Technology Fund (No. 13NM1401500 and 15DZ2252000).

\section{References}

[1] L. Chen, C. Bao, H. Yang, et al., A prototype of giant magnetoimpedance-based biosensing system for targeted detection of gastric cancer cells. Biosensors \& Bioelectronics, 2011, 26: 3246-3253.

[2] Y. Xie, X. Zhi, H. Su, et al., A novel electrochemical microfluidic chip combined with multiple biomarkers for early diagnosis of gastric cancer. Nanoscale Research Letters, 2015, 10: 477.

[3] H. Yang, M. Deng, S. Ga, et al., Capillary-driven surfaceenhanced raman scattering (sers)-based microfluidic chip for abrin detection. Nanoscale Research Letters, 2014, 9: 138.

[4] X. Zhi, M. Deng, H. Yang, et al., A novel HBV genotypes detecting system combined with microfluidic chip, loopmediated isothermal amplification and gmr sensors. Biosensors \& Bioelectronics, 2014, 54: 372-377.

[5] X. Zhi, Q. Liu, X. Zhang, et al., Quick genotyping detection of HBV by giant magnetoresistive biochip combined with PCR and line probe assay. Lab on a Chip, 2012, 12: 741-745.

[6] L.V. Panina, K. Mohri, Magneto-impedance effect in amorphous wires. Applied Physics Letters, 1994, 65: 1189.

[7] H. Yang, L. Chen, C. Lei, et al., Giant magnetoimpedance- based microchannel system for quick and parallel genotyping of human papilloma virus type 16/18. Applied Physics Letters, 2010, 97: 043702.

[8] Z. Yang, X.-C. Sun, T. Wang, et al., A giant magnetoimpedance-based biosensor for sensitive detection of escherichia coli O157:H7. Biomedical Microdevices, 2015, 17: 5.

[9] H. Hu, H. Yang, D. Li, et al., The potential of magnetic nanocluster and dual-functional protein-based strategy for noninvasive detection of HBV surface antibodies. The Analyst, 2011, 136: 679-683.

[10] J. Razumovitch, K. de Franca, F. Kehl, et al., Optimal hybridization efficiency upon immobilization of oligonucleotide double helices. The Journal of Physical Chemistry B, 2009, 113: 8383-8390.

[11] T.-H. Zheng, J.-L. Zhao, B. Guleng. Advances in molecular biomarkers for gastric cancer. Critical Reviews in Eukaryotic Gene Expression, 2015, 25: 299-305.

[12] A.P. Yang, J. Liu, H.Y. Lei, et al., CA72-4 combined with cea, CA125 and CA19-9 improves the sensitivity for the early diagnosis of gastric cancer. Clinica Chimica Acta, 2014, 437: 183-186.

[13] R. Kikuchi, Y. Abe, K. Iijima, et al., Low serum levels of pepsinogen and gastrin 17 are predictive of extensive gastric atrophy with high-risk of early gastric cancer. The Tohoku Journal of Experimental Medicine, 2011, 223: 3544.

[14] F. Nejadi-Kelarijani, G. Roshandel, S. Semnani, et al., Diagnostic values of serum levels of pepsinogens and gastrin-17 for screening gastritis and gastric cancer in a high risk area in northern iran. Asian Pacific Journal of Cancer Prevention, 2014, 15: 7433-7436.

[15] L. Wang, Y. Chang, J. Xu, et al., Predictive significance of serum level of vascular endothelial growth factor in gastric cancer patients. BioMed Research International, 2016, 2016: 8103019.

Copyright $\subset 2016$ Shan Gao, Lin Kang, Min Deng, Bin Ji, Jing Liu, Wenwen Xin, Jingjing Kang, Ping Li, Jie Gao, Jinglin Wang, and Hao Yang. This is an open-access article distributed under the terms of the Creative Commons Attribution License, which permits unrestricted use, distribution, and reproduction in any medium, provided the original author and source are credited. 